Une adaptation, pour le Canada francophone, des règles de publication de l'APA : typographie et présentation des références

French-Canadian adaptation of APA's publication rules : typography and presentation of references Una adaptación franco-canadiense de las reglas de publicación de la APA : tipografía y presentación de las referencias

\author{
Gilles Raîche et Monique Noël-Gaudreault
}

\section{Volume 35, numéro 1, 2009}

Avoir des difficultés scolaires importantes à l'école : quelles formules, quel avenir?

URI : https://id.erudit.org/iderudit/029932ar

DOI : https://doi.org/10.7202/029932ar

Aller au sommaire du numéro

Éditeur(s)

Revue des sciences de l'éducation

ISSN

0318-479X (imprimé)

1705-0065 (numérique)

Découvrir la revue

Citer cet article

Raîche, G. \& Noël-Gaudreault, M. (2009). Une adaptation, pour le Canada francophone, des règles de publication de l'APA : typographie et présentation des références. Revue des sciences de l'éducation, 35(1), 227-234.

https://doi.org/10.7202/029932ar

\section{Résumé de l'article}

Cet article a pour objectif de proposer une adaptation, pour le Canada francophone, des règles de publication de l'APA au regard de la typographie ainsi que des règles de présentation des références. Cette adaptation s'inspire des pratiques de rédaction en langue française adoptées au cours des dernières années par différents organismes et instances, ou soutenues par différents standards. Les choix effectués sont justifiés et des exemples de présentation des références, aussi bien à l'intérieur du texte qu'à la section des références, permettent d'illustrer ces choix. De plus, ces exemples montrent comment effectuer la présentation des références dans une langue autre que le français. 


\title{
Une adaptation, pour le Canada francophone, des règles de publication de l'APA: typographie et présentation des références
}

\author{
Gilles Raîche, professeur \\ Université du Québec à Montréal
}

Monique Noël-Gaudreault, professeure

Université de Montréal

\begin{abstract}
RÉSUMÉ - Cet article a pour objectif de proposer une adaptation, pour le Canada francophone, des règles de publication de l'APA au regard de la typographie ainsi que des règles de présentation des références. Cette adaptation s'inspire des pratiques de rédaction en langue française adoptées au cours des dernières années par différents organismes et instances, ou soutenues par différents standards. Les choix effectués sont justifiés et des exemples de présentation des références, aussi bien à l'intérieur du texte qu'à la section des références, permettent d'illustrer ces choix. De plus, ces exemples montrent comment effectuer la présentation des références dans une langue autre que le français.
\end{abstract}

MOTS CLÉS - références bibliographiques, règles de publication, typographie, rédaction, adaptation canadienne-française.

\section{Introduction}

La plupart des revues savantes en sciences de l'éducation ont adopté les règles de présentation de l'Association américaine de psychologie (American Psychological Association, 2001). C'est du moins ce que ces revues indiquent au regard des consignes de rédaction qu'elles donnent à leurs auteurs. Dans les faits, il s'agit très souvent plutôt d'une adaptation de ces règles. De plus, ces dernières ont été élaborées pour soutenir la publication de textes en langue anglaise. Les revues savantes en sciences de l'éducation qui publient des articles dans une autre langue que l'anglais indiquent aussi très fréquemment que leurs auteurs doivent suivre les règles de l'Association américaine de psychologie. Cependant, outre les spécificités propres à chacune des revues, les particularités de la langue de rédaction nécessitent presque toujours des adaptations importantes de ces règles. Ces adaptations concernent principalement la typographie et la présentation des références dans le texte et à la section des références.

Dans ce contexte, la Revue des sciences de l'éducation a dû prendre des décisions pour soutenir et encadrer le travail des auteurs qui lui soumettent des articles. Ces décisions ont été inspirées des pratiques de rédaction en langue française adoptées 
au cours des dernières années par différents organismes et instances ou soutenues par différents standards: revues savantes à travers leur protocole de rédaction, établissements universitaires pour encadrer la rédaction des thèses et mémoires (Boudreau, 1999; Bouthat, 1993; Faculté des études supérieures de l'Université de Montréal, 1994; Pinard, Lavoie et Delorme, 1977; Provost, Alain, Leroux et Lussier, 2006), manuel de communication écrite (Malo, 1996; Ramat, 2000) et Office de la langue française. Il s'agit de la troisième et dernière génération des règles de publication adoptées par la Revue. Celles-ci relèvent de la même logique que celles de la deuxième génération, mais elles ont été considérablement simplifiées, principalement pour faciliter l'intégration de références dans une langue différente du français.

Le présent article a pour but de présenter les choix de la Revue. En premier lieu, les éléments de la typographie de la rédaction en langue française généralement adoptés au Canada francophone seront présentés. Ensuite, nous proposerons une adaptation commentée des règles de présentation des références. Dans ce dernier cas, il s'agit clairement d'un choix de la part de la rédaction de la Revue, choix qui a pour objectif de faciliter le travail des auteurs et d'améliorer le processus rédactionnel. L'application de ces adaptations a d'ailleurs permis d'observer la diminution des délais de publications et l'amélioration de la qualité des articles publiés.

\section{2. Éléments de typographie}

Le terme typographie renvoie aussi bien à la qualité de la présentation visuelle d'un texte qu'aux conventions qui rendent la lecture du texte agréable et compréhensible. Au Québec, ces conventions sont proposées par l'Office de la langue française. Il s'avère alors que les règles de typographie adoptées au Québec francophone sont différentes, par exemple, de celles adoptées en France. À titre d'illustration, au Québec on utilise les majuscules accentuées, alors qu'en France on préfère ne pas appliquer d'accents à celles-ci.

En bonne partie, les particularités typographiques d'intérêt se rapportent à la ponctuation. C'est pourquoi ce sont celles-ci qui sont traitées dans cette section. Le tableau 1 résume les règles de typographie de l'Office de la langue française (Ramat, 2000). Avant de parcourir ce tableau, il convient toutefois de définir deux termes probablement peu familiers au lecteur. Le premier de ces termes est la notion d'une espace fine (ici, espace est de genre féminin). Une espace fine est égale à un quart de l'espace usuelle. Les logiciels de traitement de texte ne fournissent généralement pas ce type d'espace. Pour cette raison, il n'y a pas lieu d'en tenir compte et on n'utilisera pas d'espace fine dans les textes soumis à la Revue. C'est pourquoi, dans le tableau 1, il est indiqué espace fine ou rien.

Le second terme est l'espace insécable. Une espace insécable est une espace qui ne peut être coupée en fin de ligne. Une espace insécable ne peut pas être séparée d'une chaîne de caractères et ne peut donc pas être séparée de cette chaîne sur deux lignes de texte. Par exemple, une espace insécable est utilisée entre un nombre et le symbole qui le suit pour éviter que ces caractères ne se retrouvent sur deux lignes distinctes. L'espace sécable, au contraire, peut être coupée à la fin d'une ligne. Dans 
le logiciel Word, on peut insérer une espace insécable en utilisant l'option Caractères spéciaux du sous-menu Caractères spéciaux du menu Insertion. Avec Open Office, on utilisera: Insertion - Marque de formatage - Espace insécable.

Veuillez noter que la Revue, conformément à la pratique au Québec, utilise des caractères majuscules accentués.

Enfin, la virgule est utilisée comme séparateur de décimales. Il faut, dans la mesure du possible, toujours utiliser le même nombre de décimales après la virgule tout au long du texte. En général, il est préférable d'utiliser seulement deux décimales, sauf si la précision en exige plus.

Attention, le logiciel Word gère automatiquement la typographie et, dans certains cas, il pourrait être nécessaire de modifier manuellement les espaces entre les caractères. Ce pourrait être le cas, par exemple, pour les deux points «:». À noter aussi que Word et Open Office ne supportent pas naturellement l'espace fine.

Tableau 1

Espacement de la ponctuation selon Ramat (2000, p. 169)

\begin{tabular}{|c|c|c|}
\hline Ponctuation & Espace avant & Espace après \\
\hline Apostrophe dans l'élision normale: c'est & rien & rien \\
\hline Apostrophe dans l'élision exceptionnelle: L'opéra de quat' sous & rien & sécable \\
\hline Appels de note et astérisque & fine ou rien & sécable \\
\hline Arithmétique $+{ }^{*} x /=$ ou autres & sécable & sécable \\
\hline Barre oblique / ou \ & rien & rien \\
\hline Crochet ouvrant [ & sécable & rien \\
\hline Crochet fermant] & rien & sécable \\
\hline Deux-points: & insécable & sécable \\
\hline Deux-points dans les heures numériques $9: 10$ & rien & rien \\
\hline Guillemet anglais ouvrant " & sécable & rien \\
\hline Guillemet anglais fermant " & rien & sécable \\
\hline Parenthèse ouvrante ( & sécable & rien \\
\hline Parenthèse fermante ) & rien & sécable \\
\hline Point d'exclamation et point d'interrogation & fine ou rien & sécable \\
\hline Point final d'une phrase et point abréviatif & rien & sécable \\
\hline Point-virgule & fine ou rien & sécable \\
\hline Points de suspension, toujours collés entre eux... & rien & sécable \\
\hline Points elliptiques quant ils sont entre crochets [...] & rien & rien \\
\hline Pourcentage $\%$ & insécable & sécable \\
\hline Symboles $\$$ et symbole $F$ & insécable & sécable \\
\hline Symbole $\mathrm{h}$ dans une heure complexe: $10 \mathrm{~h} 25$ & insécable & insécable \\
\hline Symboles d'unités: kg, cm & insécable & sécable \\
\hline Tiret long à l'intérieur d'un texte - & sécable & sécable \\
\hline Trait d'union - & rien & rien \\
\hline Virgule & rien & sécable \\
\hline Virgule décimale & rien & rien \\
\hline
\end{tabular}




\section{Citations et références}

Plusieurs adaptations, pour le Canada francophone, des règles de présentation des références de l'Association américaine de psychologie ont été proposées, principalement à l'intérieur des établissements d'enseignement universitaires. Ces adaptations visent généralement à soutenir la rédaction des thèses de $3^{\mathrm{e}}$ cycle et des mémoires de $2^{\mathrm{e}}$ cycle. Il est à souligner que celles-ci datent quelque peu et n'ont pas toujours été modifiées depuis l'avènement de l'ordinateur. À titre d'exemple, certaines proposent encore le soulignement du titre des volumes plutôt que d'utiliser les caractères italiques, comme c'est généralement la pratique actuellement. Pour la Revue des sciences de l'éducation, nous avons retenu certaines de ces adaptations en tenant aussi compte des pratiques des revues savantes en sciences de l'éducation. Nous avons aussi pris en considération les particularités des logiciels courants de gestion bibliographique en usage et des normes informatiques pour décrire les références bibliographiques de manière à faciliter leur recherche, leur utilisation et leur partage.

\subsection{Adaptation des normes de l'American Psychological Association, APA (2001)}

Sauf exception, seules les références publiées officiellement, facilement trouvables et qui demeurent inchangées avec le temps sont référencées à l'intérieur de la Revue. Une référence correspond à un document papier ou numérisé que les lecteurs pourront obtenir et consulter en tout temps. Ainsi, les sites web, lorsqu'ils sont éphémères, ne sont pas admissibles.

La Revue a pris la décision d'utiliser une typographie spéciale pour le Canada francophone, plus spécifiquement les règles de ponctuation, aussi bien pour les références en langue française que pour celles dans une autre langue. Ainsi, pour les références dans une autre langue que le français, on utilisera le même espacement de la ponctuation qu'en français.

En ce qui concerne la citation dans le texte, il faut placer le premier auteur en ordre alphabétique croissant, ensuite par ordre croissant de l'année de publication. Il faut utiliser «et collab.» seulement lorsqu'il y a plus de deux auteurs, à moins que cela implique qu'on ne puisse distinguer deux sources. À ce moment, il faut nommer un plus grand nombre d'auteurs. Il ne faut toutefois pas utiliser «et collab.» lorsque la source est indiquée pour la première fois dans le texte. Des exemples, accompagnés de précisions supplémentaires, sont présentés au tableau 2.

En langue française, un éditeur désigne la maison d'édition qui produit le document recensé et non pas la personne qui a dirigé la publication de celui-ci. Pour cette raison, il faut remplacer (Éd.) par (Dir.) lorsqu'on désire spécifier cette fonction. Ce sera le cas, par exemple, pour un chapitre de livre.

À moins qu'on désire faire référence à une source d'information dans son entièreté, il est toujours préférable d'indiquer dans le texte la ou les pages d'où proviennent les informations. Le lecteur pourra les retrouver plus facilement, tout comme l'auteur lui-même, sans avoir à relire le document au complet. 
Il faut aussi éviter d'utiliser des acronymes pour référer à un organisme qui serait l'auteur de la référence. Il faut écrire le nom de l'organisme au complet et s'assurer que sa formulation est exactement la même dans le texte et à la section des références. Cette consigne est nécessaire, car les lecteurs de la Revue proviennent de différents milieux et de tous les coins de la planète. Les acronymes en jeu peuvent n'avoir aucune signification en dehors de l'environnement immédiat de l'auteur. Le tableau 2 donne des exemples, parmi les plus fréquents, de présentation de citation dans le texte.

Tableau 2

Citations des auteurs dans le texte (adaptation de l'American Psychological Association, 2001)

\begin{tabular}{ll}
\hline Particularités & Exemples \\
\hline Format général de citation intégrée au texte. & $\begin{array}{l}\text { Comme le soulignent Mialaret (2003) ainsi que } \\
\text { Kalubi, Detraux et Larivée (1996, 2000a, 2000b) ... }\end{array}$
\end{tabular}

Format général de citation non intégrée au texte. Plusieurs auteurs ont abordé le sujet (Kalubi, Detraux et Larivée, 1996, 2000a, 2000b; Mialaret, 2003) ...

Format à utiliser lorsque nous n'avons pas consulté la source primaire et que nous ne retenons que l'interprétation obtenue d'une source secondaire.

van Gennep (1960: voir Richard, 1998) a traité abondamment de ce sujet ...

Ce sujet a été traité à fond par les auteurs (van Gennep, 1960: voir Richard, 1998)

L'indication des pages ou des chapitres consultés n'est pas obligatoire, mais la pratique se (Mialaret, 2003, chap. 10; Simon et ForgetteGiroux, 2003, p. 276-277) ... généralise de plus en plus. Elle sera fort utile pour retrouver l'information plus tard.

On peut aussi consulter les informations fournies par l'American Psychological Association (2001, chap. 3).

On utilise la forme McCaffrey et collab. (2005) McCaffrey et collab. (2005) ou (McCaffrey et collab., 2005) lorsqu'il n'y a pas (McCaffrey et collab., 2005) risque d'ambiguïté, que c'est au moins la seconde fois que la source est indiquée et qu'il $y$ a plus de deux auteurs (American Psychological Association, 2001, p. 208).

Les tableaux 3 et 4 donnent des exemples de présentation de divers types de références fréquemment utilisées. Plusieurs situations sont prises en compte. Ainsi, on remarquera des exemples du même type de référence aussi bien en langue française qu'en langue anglaise. On remarquera aussi des variations en ce qui a trait au nombre d'auteurs et au numéro d'édition d'un volume. Il est à noter que nous avons pris la décision de conserver le et, non pas le and, et cela, uniquement dans le but de faciliter le traitement des références dans les logiciels de citation tels que End Note.

Seuls les types de citations et de références les plus fréquemment utilisés sont présentés ici en exemple. Si vous êtes confrontés à certaines autres particularités, consultez le manuel de l'APA (2001). Si le type de référence ne correspond pas à un des modèles présentés aux tableaux 3 ou 4, il faut généralement utiliser le 
modèle qui est associé à un rapport. Les références utilisées aux fins d'une métaanalyse, conformément à la pratique, sont tout simplement précédées par un astérisque.

\section{Tableau 3}

Les références à l'intérieur de revues ou de volumes (adaptation de l'American Psychological Association, 2001)

\begin{tabular}{|c|c|}
\hline Particularités & Exemples \\
\hline Revue 1 & $\begin{array}{l}\text { Kalubi, J.-C., Detraux, J.-J. et Larivée, S. J. (2006). Participation des familles en } \\
\text { contexte d'inclusion sociale: une contribution en faveur de la bientraitance des } \\
\text { élèves. Revue des sciences de l'éducation, 32(3), 517-524. }\end{array}$ \\
\hline Revue 2 & $\begin{array}{l}\text { Jenkins, J. R., Dale, P. S., Mills, P. E., Cole, K. N., Pious, C. et Ronk, J. (2006). How } \\
\text { special education preschool graduate finish: status at } 19 \text { years of age. American } \\
\text { educational research journal, } 43(4), 737-781 .\end{array}$ \\
\hline Volume 1 & $\begin{array}{l}\text { Mialaret, G. (2003). Propos impertinents sur l'éducation. Paris, France: Presses } \\
\text { universitaires de France. }\end{array}$ \\
\hline Volume 2 & $\begin{array}{l}\text { Van Gennep, A. (1960). The rites of passage ( } 2^{\mathrm{e}} \text { édition). Chicago, Illinois: The } \\
\text { University of Chicago Press. }\end{array}$ \\
\hline $\begin{array}{l}\text { Section d'un } \\
\text { volume } 1\end{array}$ & $\begin{array}{l}\text { Raîche, G. et Blais, J.-G. (2003). Efficacité du dépistage des étudiantes et des } \\
\text { étudiants qui cherchent à obtenir un résultat faible au test de classement en anglais, } \\
\text { langue seconde, au collégial. Dans J.-G. Blais et G. Raîche (Dir.): Regards sur la } \\
\text { modélisation de la mesure en éducation et en sciences sociales. Sainte-Foy, Québec: } \\
\text { Presses de l'Université Laval. }\end{array}$ \\
\hline $\begin{array}{l}\text { Section d'un } \\
\text { volume } 2\end{array}$ & $\begin{array}{l}\text { Simon, M. et Forgette-Giroux, R. (2003). Évaluer pour informer : l'utilisation du dossier } \\
\text { d'apprentissage. Dans M. D. Laurier (Dir.): Évaluation et communication - } \\
\text { De l'évaluation formative à l'évaluation informative. Montréal, Québec: Éditions } \\
\text { Quebecor. }\end{array}$ \\
\hline $\begin{array}{l}\text { Section d'un } \\
\text { volume } 3\end{array}$ & $\begin{array}{l}\text { Newstead, S., Bradon, P., Handley, S., Evans, J. et Dennis, 1. (2002). Using the } \\
\text { psychology of reasoning to predict the difficulty of analytical reasoning problems. } \\
\text { Dans S. H. Irvine et P. C. Kyllonen (Dir.): Item generation for test development. } \\
\text { Mahwah, New Jersey: Lawrence Erlbaum Associates. }\end{array}$ \\
\hline
\end{tabular}

Tableau 4

Les références de types divers (adaptation de l'American Psychological Association, 2001)

\begin{tabular}{|c|c|}
\hline Particularités & Exemples \\
\hline Actes 1 & $\begin{array}{l}\text { Lebrun, N., Verreault, L. et Perreault, D. (2007). Intégration et expérimentation du } \\
\text { programme de développement des compétences informationnelles dans le cadre de } \\
\text { la formation des étudiants maîtres à l'UQAM. Actes du } 24^{e} \text { congrès de l'AIPU. } \\
\text { Montréal, Québec: Association internationale de pédagogie universitaire. }\end{array}$ \\
\hline Actes 2 & $\begin{array}{l}\text { Sheng, Y., Flournoy, N. et Osterlind, S. J. (2007). Up-and-down procedures for } \\
\text { approximating optimal test designs using person-response functions. Proceedings of } \\
\text { the GMAC computerized adaptive testing conference. Minneapolis, Minnesota: } \\
\text { Graduate Management Admission Council. }\end{array}$ \\
\hline
\end{tabular}


Thèse 1 Richard, J.-F. (1998). La mesure et l'évaluation de la performance en jeux et sports collectifs: la participation des élèves du primaire dans une perspective d'évaluation authentique. Thèse de doctorat inédite, Université Laval, Sainte-Foy.

Thèse 2 Parent, C. (1986). The assessment of academic self-concept. Unpublished doctoral dissertation, University of Toronto, Toronto.

Mémoire $\quad$ Banville, D. (1994). Le passage du primaire au secondaire sur le plan du contenu enseigné en éducation physique. Mémoire de maîtrise inédit, Université Laval, Sainte-Foy.

Banque ERIC Karsenti, T. et Thibert, G. (1998). The interaction between teaching practices and the change in motivation of elementary-school children. Paper presented at the annual conference of the American Educational Research Association (AERA), San Diego, California. (Document ERIC n ED420397).

Rapport 1 Ministère de l'Éducation du Québec (2001). Programme de formation de l'école québécoise. Éducation préscolaire - Enseignement primaire. Québec, Québec: Gouvernement du Québec.

Rapport 2 Magis, D. (2006). A new use of likelihood-ratio statistic to detect decisive subgroups of populations in testing homogeneity of proportions. Working paper $n^{\circ} 06.001$. Liège, Belgium : University of Liège, Department of mathematics.

Journal Castonguay, A. (2008, 18 septembre). À 21 ans, les études inachevées, Nicolas Dufour pourrait se retrouver sur les banquettes du Parlement. Le Devoir, A6.

\section{Conclusion}

Cet article avait pour objectif de décrire et de justifier les règles de publication adoptées par la Revue des sciences de l'éducation. Plus spécifiquement, ce sont les règles typographiques et de présentation des références qui étaient ciblées. Il faut espérer que cet exercice pourra faciliter le travail des auteurs de la Revue, ainsi que soutenir et accélérer le travail de rédaction. L'utilisation de cette adaptation, pour le Canada francophone, à l'intérieur d'autres revues savantes ou publications est aussi encouragée. Enfin, les règles proposées ici pourront se transformer au fil des ans pour tenir compte des changements dans les pratiques de publication et pour améliorer l'efficacité de la consultation des références par les lecteurs.

ENGLISH TITLE - French-Canadian adaptation of APA's publication rules : typography and presentation of references

SUMmARY - The objective of this article is to propose a French-Canadian adaptation of APA's publication rules. More specifically, typography and presentation of references are of concern here. The adaptation draws its inspiration from French edition practices adopted by different organisms and associations or from support by different standards. Choices are justified and examples of reference presentation, within the text and in the reference section, illustrate these choices. Also, these examples show how to present references in a language other than French.

KEY WORDS - references, publication rules, typography, edition, French-Canadian adaptation. 
TítULO EN ESPAÑOL - Una adaptación franco-canadiense de las reglas de publicación de la APA: tipografía y presentación de las referencias

RESUMEN - El objetivo del presente artículo es proponer una adaptación franco-canadiense de las reglas de publicación de la APA en cuanto a la tipografía así como de las reglas de presentación de las referencias. Esta adaptación se inspira de las prácticas de redacción en lengua francesa adoptadas durante los últimos años por diversos organismos e instancias, o sostenidas por diferentes estándares. Se justifican las opciones elegidas y se proporcionan ejemplos de presentación de las referencias, tanto en el texto como en la sección de las referencias que permiten ilustrar estas opciones. Además, estos ejemplos muestran cómo realizar la presentación de las referencias en otro idioma que el francés.

PALABRAS CLAVES - referencias bibliográficas, reglas de publicación, tipografía, redacción, adaptación franco-canadiense.

\section{Références}

American Psychological Association (2001). Publication Manual of the American Psychological Association. Washington, District of Columbia: American Psychological Association, APA.

Boudreau, B. (1999). Guide de présentation d'une thèse. Moncton, Nouveau-Brunswick: Université de Moncton.

Bouthat, C. (1993). Guide de présentation des mémoires et des thèses. Montréal, Québec: Université du Québec à Montréal.

Faculté des études supérieures de l'Université de Montréal (1994). Procédure d'acceptation et guide de présentation des mémoires et des thèses. Montréal, Québec: Université de Montréal.

Malo, M. (1996). Guide de la communication écrite au cégep, à l'université et en entreprise. Montréal, Québec: Québec/Amérique.

Pinard, A., Lavoie, G. et Delorme, A. (1977). La présentation des thèses et des rapports scientifiques - Normes et exemples ( $3^{\text {e }}$ édition). Montréal, Québec: Institut de recherches psychologiques.

Provost, M. A., Alain, M., Leroux, Y. et Lussier, Y. (2006) Normes de présentation d'un travail de recherche. Trois-Rivières, Québec: Éditions SMG.

Ramat, A. (2000). Le Ramat de la typographie. Saint-Laurent, Québec: Aurèle Ramat éditeur. 